\title{
Echocardiographic assessment of right ventricular function: current clinical practice
}

\author{
Matthias Schneider ${ }^{1} \cdot$ Stefan Aschauer ${ }^{1} \cdot$ Julia Mascherbauer $^{1} \cdot$ Hong Ran $^{2} \cdot$ Christina Binder $^{1} \cdot$ Irene Lang $^{1}$. \\ Georg Goliasch ${ }^{1} \cdot$ Thomas Binder $^{1}$
}

Received: 30 June 2018 / Accepted: 26 July 2018 / Published online: 6 September 2018

(c) The Author(s) 2018

\begin{abstract}
Echocardiographic evaluation of right ventricular (RV) function is a challenge due to the complex anatomy of the RV. Several transthoracic echocardiographic methods have been suggested for the quantification of RV function. However, many of the parameters are time consuming and need dedicated hardware and software. We suspected that the majority of the established markers are not used on a wide basis. In a multinational online survey, we evaluated the use of current clinical standards for the quantification of RV function in clinical practice. Through the network of an Ultrasound Online Teaching Platform, echocardiographers were invited to participate in an open online survey. The participants were asked about the parameters (eyeballing, TAPSE, $\mathrm{S}^{\prime}$, fractional area change, RIMP, 3D-EF, dp/dt, longitudinal strain) they used in clinical practice. A total of 1150 participants from 109 countries completed the survey. Only eyeballing (72\%), TAPSE (69\%), and S' (31\%) were commonly used in clinical routine. These methods were applied significantly less common in low-income economies when compared to high-income economies. Twenty-three percent of all participants stated to rely on eyeballing only, when evaluating RV function in clinical routine. New technologies, such as global longitudinal strain (3\%) and 3D echocardiography (1\%) were rarely applied independent of region and economic strength. Eyeballing and TAPSE are the most widely used methods in echocardiography for the assessment of RV function. Although advanced parameters such as longitudinal strain and 3D echocardiography were shown to be highly accurate, they are rarely used in clinical routine.
\end{abstract}

Keywords Right ventricular function · Transthoracic echocardiography $\cdot$ TAPSE $\cdot$ Eyeballing

\section{Introduction}

Correct assessment of right ventricular (RV) function is an essential part of every transthoracic echocardiographic (TTE) examination. It plays an important role in the diagnosis and management of many diseases and conditions.

TTE evaluation of RV function (RVF) remains challenging due to the complex anatomy of the RV. In contrast to the left ventricle, there is no exact geometric model for the $\mathrm{RV}$ that would permit volumetric measurements based on 2D echocardiographic images. Right ventricular ejection fraction (RVEF) derived from cardiac magnetic resonance

Thomas Binder

thomas.binder@meduniwien.ac.at

1 Department of Internal Medicine II, Medical University of Vienna, Waehringer Guertel 18-20, 1090 Vienna, Austria

2 Department of Echocardiography, Nanjing First Hospital Affiliated to Nanjing Medical University, Nanjing, China imaging (MRI) remains the gold standard for functional RV assessment.

Several parameters have been suggested for echocardiographic evaluation of the RV. The simplest and most commonly used parameter is visual assessment. The reliability of visual quantification has been evaluated in previous studies $[1,2]$; the authors concluded that eyeballing alone is insufficient to quantify RV function. However, factors influencing visual assessment and its actual use in various parts of the world remain ambiguous.

Guidelines for TTE assessment of the right heart demand the use of at least one quantitative parameter in addition to visual gradation [3]. Several studies have demonstrated the value of tricuspid annular plane systolic excursion (TAPSE) [4-6], tissue Doppler imaging of the basal free lateral wall of the RV ( $\left.\mathrm{S}^{\prime}\right)$ [7], longitudinal strain of the free lateral wall of the right ventricle (RV-GLS) [8-10], fractional area change (FAC) [11-14], right ventricular myocardial performance index (RIMP) [7, 15], and the rate of pressure rise in the RV 
(dp/dt) [16]. Furthermore, three-dimensional echocardiography allows an exact and reproducible estimation of volume and ejection fraction of the RV [17-20].

However, we lack knowledge about the gap between guideline recommendations and the actual assessment of $\mathrm{RV}$ function in clinical practice. For the present study, we performed a multinational online survey to determine those methods most frequently used by echocardiographers for the quantification of RV function.

\section{Materials and methods}

Through the network of an Ultrasound Teaching Platform (123sonography.com), echocardiographers were invited to participate in an open online survey. All answers received between April 1 and July 312017 were analyzed. The study was approved by the ethics committee of the Medical University of Vienna (EK \#1288/2016). The study protocol is in conformity with the ethical guidelines of the Declaration of Helsinki.

\section{Questionnaire design}

The participant's name and the name of the institution were not included in the questionnaire. Baseline demographic data were obtained, such as age, the country in which participants practiced, profession, work setting (university hospital, hospital, private clinic/private office, other), and experience in TTE.

Participants were asked about the parameters they used for the assessment of right ventricular function. They answered two questions, (1) which methods of RV quantification they apply occasionally as part of their individual diagnostic repertoire, and (2) which methods they apply in daily clinical routine on most of their examinations.

Common methods (eyeballing, TAPSE, S', FAC, RIMP, 3D-EF, dp/dt, and RV-GLS) were given as possible answers in a multiple-choice format. When applicable, the respondents could mention other techniques in a free-response box.

\section{Objectives}

The main objective of this study was to determine which methods for the evaluation of right ventricular function are applied in daily clinical practice in a real-life sample of echocardiographers from all over the world.

\section{Different regions of the world and differences between low and high income economies}

The World Bank divides the countries of the world into four income classes depending on the gross national income per capita ranking (GNI). Low income economies were defined as those with a GNI of $\$ 1005$ or less in 2016. Lower middle-income economies were defined as those with a GNI between $\$ 1006$ and \$3955. Upper middle-income economies were defined as those with a GNI between $\$ 3956$ and $\$ 12,235$. High-income economies were defined as those with a GNI of above $\$ 12,235$ [21].

According to the World Bank, we divided the world into the following seven regions to analyze regional differences: East Asia and Pacific, Europe and Central Asia, Latin America and the Caribbean, Middle East and North Africa, North America, South Asia, and Sub-Saharan Africa.

\section{Statistical analysis}

Categorical data are presented as absolute numbers and percentages. Comparison of descriptive data between groups was performed using $\chi^{2}$ test and Fisher's exact test as appropriate. A $p$ value of $<0.05$ was considered statistically significant. SPSS Version 24 (IBM SPSS, USA) was used for all analyses.

\section{Results}

A total of 1150 participants from 109 countries completed the survey. Sixty-three percent of the participants were from high-income economies, $37 \%$ were from lower income economies (including low income, lower middle-income, and higher middle-income). Thirty-nine percent were cardiologists, $23 \%$ were sonographers, and 19\% internal medicine specialists. Twenty-eight percent worked in university hospitals and $48 \%$ in non-tertiary hospital settings. Nineteen percent of the participants described themselves as beginners in echocardiography, $45 \%$ as moderately advanced, $29 \%$ as advanced, and $7 \%$ as experts. Eleven percent of the participants worked in the United States of America, 7\% in Germany, 5\% in Austria, 5\% in India, and 5\% in the United Kingdom. Full descriptive statistics are shown in Table 1.

\section{Individual diagnostic repertoire}

Seventy-nine percent of the participants $(n=906)$ stated that they used eyeballing to assess right ventricular function. TAPSE was used by $82 \%$ of the participants, $S^{\prime}$ by $48 \%$, FAC by $26 \%$, and RV-GLS by $11 \%$. RIMP, 3D echocardiography, and $\mathrm{dp} / \mathrm{dt}$ was used by 7,4 , and $6 \%$ respectively. Twenty percent of the participants used one method (including eyeballing) to assess right ventricular function, $29 \%$ used two methods, and $51 \%$ three or more methods. 
Table 1 Baseline characteristics of the study population $(n=1150)$

\begin{tabular}{|c|c|}
\hline Baseline characteristics & All \\
\hline Number of participants & 1150 \\
\hline \multicolumn{2}{|l|}{ Age (years) } \\
\hline$<30, \mathrm{n}(\%)$ & $119(10)$ \\
\hline $30-39, \mathrm{n}(\%)$ & $418(36)$ \\
\hline $40-49, \mathrm{n}(\%)$ & $298(26)$ \\
\hline $50-59, \mathrm{n}(\%)$ & $200(17)$ \\
\hline $60-69, \mathrm{n}(\%)$ & $105(9)$ \\
\hline$>69, \mathrm{n}(\%)$ & $10(1)$ \\
\hline \multicolumn{2}{|l|}{ Profession } \\
\hline Sonographer, n (\%) & $264(23)$ \\
\hline $\mathrm{MD}$, internal medicine, $\mathrm{n}(\%)$ & $214(19)$ \\
\hline MD, cardiologist, n (\%) & $447(39)$ \\
\hline $\mathrm{MD}$, anesthesiologist, $\mathrm{n}(\%)$ & $53(5)$ \\
\hline MD, emergency n (\%) & $27(2)$ \\
\hline $\mathrm{MD}$, intensive care, $\mathrm{n}(\%)$ & $51(4)$ \\
\hline MD, other doctor, n (\%) & $60(5)$ \\
\hline Other, n (\%) & $34(3)$ \\
\hline \multicolumn{2}{|l|}{ Work setting } \\
\hline University hospital, n (\%) & $322(28)$ \\
\hline Hospital, n (\%) & $549(48)$ \\
\hline Private practice, n (\%) & $242(21)$ \\
\hline Other, n (\%) & $37(3)$ \\
\hline \multicolumn{2}{|l|}{ Level of expertise } \\
\hline Beginner, n (\%) & $224(19)$ \\
\hline Moderately advanced, n (\%) & $519(45)$ \\
\hline Advanced, n (\%) & $329(29)$ \\
\hline Expert, n (\%) & $78(7)$ \\
\hline \multicolumn{2}{|l|}{ Number of echos performed } \\
\hline$<100, \mathrm{n}(\%)$ & $164(14)$ \\
\hline $100-500, \mathrm{n}(\%)$ & $232(20)$ \\
\hline $501-1000, \mathrm{n}(\%)$ & $178(15)$ \\
\hline $1001-5000, \mathrm{n}(\%)$ & $258(22)$ \\
\hline$>5000, \mathrm{n}(\%)$ & $318(28)$ \\
\hline \multicolumn{2}{|l|}{ Country } \\
\hline USA, n (\%) & $127(11)$ \\
\hline Germany, n (\%) & $85(7)$ \\
\hline Austria, n (\%) & $61(5)$ \\
\hline India, n (\%) & $56(5)$ \\
\hline United Kingdom, n (\%) & $52(5)$ \\
\hline Italy, n (\%) & $50(4)$ \\
\hline Poland, n (\%) & $37(4)$ \\
\hline Australia, n (\%) & $35(4)$ \\
\hline Sweden, n (\%) & $34(3)$ \\
\hline Greece, n (\%) & $33(3)$ \\
\hline Other countries, n (\%) & $580(50)$ \\
\hline
\end{tabular}

$M D$ medical doctor
Methods regularly used in daily clinical routine

Only eyeballing (72\%), TAPSE (69\%), and $\mathrm{S}^{\prime}(31 \%)$ were mentioned as frequently used methods. Thirty-nine percent of the participants applied only one method (including eyeballing) to assess right ventricular function in daily clinical practice, $35 \%$ used two methods, and $26 \%$ more than two methods. Eyeballing was applied as a single parameter by $23 \%$ of the participants (Table 2; Fig. 1).

\section{University versus non-university setting}

Significant differences were registered between those working in a university setting and those in non-university settings. TAPSE $(p=0.001), S^{\prime}(p=0.01)$, FAC $(p=0.004)$, 3D-EF $(p<0.001)$, and RV-GLS $(p<0.001)$ were significantly more often mentioned as part of the individual diagnostic repertoire in the university setting. Those practicing at universities also used significantly $(\mathrm{p}<0.001)$ more methods to assess RVF. However, the analysis of the respondents' answers concerning daily clinical routine revealed no significant differences between the two settings (see Table 2).

\section{Experience with pulmonary hypertension patients}

Sixty percent of the respondents stated that they regularly examined patients with severe pulmonary hypertension $(\mathrm{PH})$. A significant $(\mathrm{p}<0.001)$ difference was registered between the PH-center and the non-PH-center group with regard to the number of methods used in clinical routine. At PH centers, $31 \%$ of the respondents reported the use of three or more parameters (including eyeballing) to assess right ventricular function in clinical routine, as opposed to $16 \%$ at non-PH-centers. All imaging parameters were used more frequently at $\mathrm{PH}$ centers; the difference was statistically significant for $\mathrm{S}^{\prime}(\mathrm{p}<0.001)$, FAC $(\mathrm{p}=0.001)$, and RIMP $(p=0.006)$. Full data about imaging at PH centers are summarized in Table 2.

\section{Cardiologists versus non-cardiologists}

Eyeballing was used significantly more frequently by noncardiologists, while TAPSE was used significantly more often by cardiologists. The differences in all other parameters did not reach statistical significance (data not shown).

\section{Differences between high income and low income economies}

Results were compared between high income economies on the one hand and low income, lower middle-income, and upper middle-income on the other hand. There were significant differences between the two groups. While eyeballing, TAPSE, 
Table 2 Parameters used in daily clinical routine for evaluation of right ventricular function

\begin{tabular}{|c|c|c|c|c|}
\hline Methods applied in daily clinical routine & Total & University hospital & Others & $\mathrm{p}$ value \\
\hline Eyeballing, n (\%) & $832(72)$ & $231(72)$ & $601(73)$ & 0.774 \\
\hline TAPSE, n (\%) & $798(69)$ & $241(75)$ & $557(67)$ & 0.012 \\
\hline $\mathrm{S}^{\prime}, \mathrm{n}(\%)$ & $353(31)$ & $108(34)$ & $245(3)$ & 0.192 \\
\hline $\mathrm{FAC}, \mathrm{n}(\%)$ & $104(9)$ & $35(11)$ & $69(8)$ & 0.178 \\
\hline RIMP, n (\%) & $20(2)$ & $7(2)$ & $13(2)$ & 0.482 \\
\hline 3D-EF, n (\%) & $13(1)$ & $5(2)$ & $8(1)$ & 0.398 \\
\hline $\mathrm{dp} / \mathrm{dt}, \mathrm{n}(\%)$ & $26(2)$ & $6(2)$ & $20(2)$ & 0.572 \\
\hline GLS-RV, n (\%) & $29(3)$ & $12(4)$ & $17(2)$ & 0.104 \\
\hline Methods applied in daily clinical routine & All & PH-center & Non-PH-center & $\mathrm{p}$ value \\
\hline Eyeballing, n (\%) & $832(72)$ & $503(73)$ & $329(72)$ & 0.679 \\
\hline TAPSE, n (\%) & 798 (69) & $488(71)$ & $310(68)$ & 0.266 \\
\hline $\mathrm{S}^{\prime}, \mathrm{n}(\%)$ & $353(31)$ & $245(35)$ & $108(24)$ & $<0.001$ \\
\hline FAC, n (\%) & $104(9)$ & 79 (11) & $25(5)$ & 0.001 \\
\hline RIMP, n (\%) & $20(2)$ & $18(3)$ & $2(0)$ & 0.006 \\
\hline 3D-EF, n (\%) & $13(1)$ & $10(2)$ & $3(1)$ & 0.213 \\
\hline $\mathrm{dp} / \mathrm{dt}, \mathrm{n}(\%)$ & $26(2)$ & $17(2)$ & $9(2)$ & 0.577 \\
\hline GLS-RV, n (\%) & $29(3)$ & $20(3)$ & $9(2)$ & 0.323 \\
\hline
\end{tabular}

Comparison between different hospital settings

TAPSE tricuspid annular plane systolic excursion, $S^{\prime}$ tissue Doppler velocity of the basal free lateral wall of the right ventricle, $F A C$ fractional area change, RIMP right ventricular myocardial performance index, $3 D$ $E F 3 \mathrm{D}$ ejection fraction, $D p / d t$ the rate of pressure rise in the RV, $G L S-R V$ global longitudinal strain of the free wall of the right ventricle

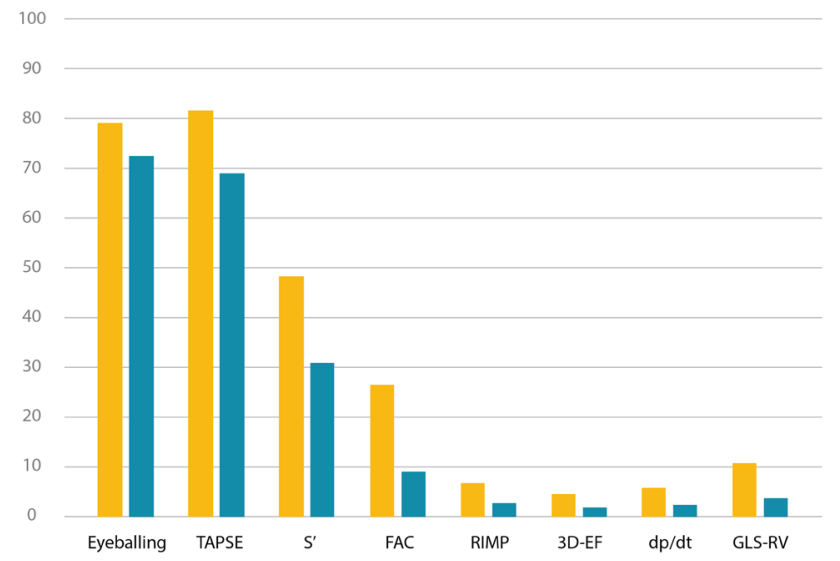

Fig. 1 Methods used for right ventricular functional assessment: overall (yellow bar) and daily clinical routine (blue bar). TAPSE tricuspid annular plane systolic excursion, $S^{\prime}$ tissue Doppler velocity of the basal free lateral wall of the right ventricle, $F A C$ fractional area change, RIMP right ventricular myocardial performance index, $3 D$ $E F$ 3D ejection fraction, $D p / d t$ the rate of pressure rise in the RV, $G L S-R V$ global longitudinal strain of the free wall of the right ventricle, $Y$-axis percent of the participants
$\mathrm{S}^{\prime}$, and FAC are the four most commonly applied parameters in both groups, all of the methods were applied significantly more often in the high-income economies as opposed to the lower income economies (data not shown).

\section{Differences between regions of the world}

Eyeballing (72\%) and TAPSE (69\%) are the most commonly applied parameters in all regions of the world. TAPSE is used less frequently in the Middle East and North Africa (49\%) and in Sub-Saharan Africa (44\%), $\mathrm{S}^{\prime}$ is used less frequently in South Asia (17\%) and in Sub-Saharan Africa (13\%). Furthermore, there were differences in the number of parameters applied for RV function gradation. Eyeballing as a single parameter was used in less than $20 \%$ of the participants from East Asia and Pacific, Europe and Central Asia, and Latin America and the Caribbean, and by $30 \%$ of those from North America, by $37 \%$ by those from the Middle East and North Africa, by $28 \%$ of the participants from South Asia, and by $48 \%$ of those from Sub-Saharan Africa (Fig. 2). 


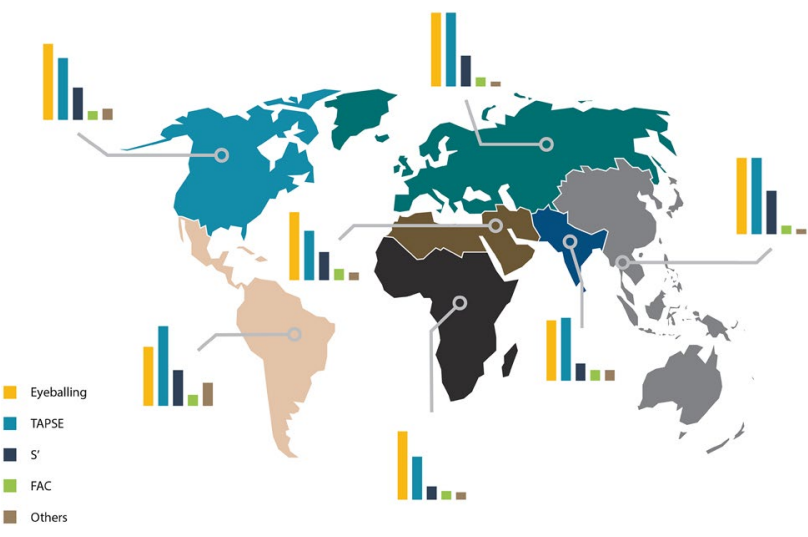

Fig. 2 Regional differences in right ventricular functional assessment. TAPSE tricuspid annular plane systolic excursion, $S^{\prime}$ tissue Doppler velocity of the basal free lateral wall of the right ventricle, $F A C$ fractional area change

\section{Discussion}

To our knowledge this is the first evaluation of clinical practice concerning the assessment of right ventricular function by echocardiography in a large group of health care professionals (medical doctors and sonographers) on a global scale. Eyeballing (79\%) and TAPSE (82\%) were the most commonly used parameters, followed by the tissue Doppler parameter $\mathrm{S}^{\prime}$, which was applied by $31 \%$ of respondents. All other parameters were used infrequently. Notably, the novel parameters such as RV-GLS and 3D echocardiography have not yet been established in clinical routine-neither in the academic setting nor in non-tertiary hospitals. Guidelines recommend that, in addition to eyeballing, at least one other parameter should be used to assess right ventricular function. Eyeballing alone is not sufficient for the quantification of right ventricular function [1, 2]. However, only $77 \%$ of our respondents adhered to this recommendation.

This gap between current scientific knowledge and actual real-world echocardiography deserves attention and should be addressed by the echocardiographic community.

\section{Tricuspid annular plane systolic excursion and tissue Doppler imaging}

TAPSE and $\mathrm{S}^{\prime}$, as measures of longitudinal motion of the basal lateral free wall of the RV, have several limitations. They only reflect the motion of the basal segments of the $\mathrm{RV}$ and may lead to a significant over- or underestimation of right ventricular function in the presence of regional differences in contraction. Displacement of the apex, resulting in passive motion of basal right ventricular segments, may result in the overestimation of RVF using TAPSE. TAPSE does not reflect true right ventricular function in many clinical conditions, including patients who have undergone cardiac surgery, those with Tetralogy of Fallot, and those with tricuspid regurgitation [22-25]. Apart from eyeballing, TAPSE $(69 \%)$ and $\mathrm{S}^{\prime}(31 \%)$ are the most commonly used parameters for RVF gradation. This may be due to the fact, that TAPSE and $\mathrm{S}^{\prime}$ are simple parameters, can be applied rapidly, and do not require sophisticated technology.

\section{Fractional area change}

FAC is a quantitative parameter that reflects volume change during contraction. Nine percent of our respondents used this parameter in clinical routine. The meager use of this procedure is surprising in view of the fact that FAC can be measured with every echo machine; no Doppler recordings or additional software is needed. Moreover, the clinical value of the parameter has been confirmed in several studies [11-14].

Eleven percent of those who worked at $\mathrm{PH}$ centers used FAC in clinical routine, as opposed to $5 \%$ at non-PH centers $(p=0.001)$. The significantly more frequent use of FAC at $\mathrm{PH}$ centers indicates the valuable additional information provided by this method, especially in patients with severely dilated right ventricles and reduced FAC, but preserved longitudinal contraction. The rare use of the method at non-PH centers may be explained by the additional time taken to perform the procedure, and uncertainty about correct tracing of the right ventricular endocardial border.

\section{Myocardial performance index and the rate of pressure rise in the right ventricle}

RIMP and dP/dt reliably predict reduced right ventricular function. Among our echocardiographers, both methods were used by a mere $2 \%$ in clinical practice. Dp/dt and RIMP require the recording of special Doppler signals which might not be a part of the standard imaging protocol at many echocardiography laboratories. Acquisition is not as straightforward as the simple measurement of distance (TAPSE), area $(\mathrm{FAC})$, or peak velocity $\left(\mathrm{S}^{\prime}\right)$. The infrequent use may be related to difficulties in understanding physiologic and pathologic principles as well as the additional time taken for the assessment.

\section{New technologies}

As expected, new and advanced parameters of RV quantification are used more frequently in the university setting than in the non-university setting. However, the difference did not persist in daily clinical routine.

Longitudinal strain of the free lateral wall of the RV is a promising new technology to asses RVF. It measures the deformation of the myocardium and permits regional as well as global assessment of the free lateral wall of the 
right ventricle. RV longitudinal strain is occasionally used by $11 \%$, and regularly used by $3 \%$ in clinical routine. Strain analysis requires dedicated software, good image quality, ECG tracing, and additional time for analysis. The method is easy to use but expensive. Our data showed that although strain analysis has been available for several years, it is rarely used in daily clinical practice.

3D volume assessment yields end-diastolic and end-systolic volumes as well as ejection fraction and stroke volume. It is not affected by regional variations in RV contractility or passive right heart motion. 3D echocardiography was occasionally used by $4 \%$ of the respondents and regularly used by $1 \%$ in clinical routine. 3D echocardiography requires dedicated software and hardware, which may not be available at many centers. Advanced and expensive imaging systems are available at university hospitals and usually do not exist in smaller hospitals or medical offices. Thus, it was no surprise to note that $3 \mathrm{D}$ echocardiography is available more frequently in university hospitals than in other settings ( 8 vs. $3 \%, \mathrm{p}<0.001)$. Time and experience also play a role. A multi-beat $3 \mathrm{D}$ dataset of the right ventricle including offline analysis requires significantly more time than TAPSE and $\mathrm{S}^{\prime}$ and - in addition - the skill to acquire loops with very good image quality. It is used significantly less often by beginners than by advanced echocardiographers.

RV-GLS and volume assessment of the RV by 3D echocardiography are promising new tools for the assessment of right ventricular function. The present investigation showed that significant effort will be needed in the future to implement these methods in daily routine. Echocardiographers will have to be trained in, and convinced of, the use of these parameters. Recent promising developments in 3D technology have accelerated and improved the acquisition and interpretation of RV-3D datasets by introducing a single-beat method for RV volume analysis [26]. The industry should now focus on further simplification of the new methods and the development of affordable algorithms.

\section{Differences between regions of the world and different levels of economic strength}

Eyeballing and TAPSE are the most commonly applied parameters in all regions of the world. TAPSE is used less frequently in the Middle East and North Africa and in SubSaharan Africa, $\mathrm{S}^{\prime}$ is used less frequently in South Asia and in Sub-Saharan Africa.

There were significant differences between high-income and lower income economies. While eyeballing, TAPSE, S', and FAC are the four most commonly applied parameters in both groups, all of the methods were applied significantly more often in the high-income economies as opposed to the low-income economies. As TAPSE and FAC are parameters solely based on $2 \mathrm{D}$ measurements which can be performed by every echo machine, higher costs for more sophisticated imaging systems do not explain the observed differences. The differences can be explained through less reachable training in echocardiography, different health care systems with less capacities for the diagnosis and treatment of complex diseases and therefore more focused examinations.

\section{Limitations}

Data were collected anonymously through an online survey. Therefore, accuracy of the individual responses could not be verified; we had to rely on the honesty of the participants. However, it may be assumed that the results overestimate true clinical practice and not vice versa. The anonymous nature of the survey permitted a large number of persons to participate in the study. The study participants were recruited from the users of an online teaching platform, which may have signified a selection bias in favor of echocardiographers interested in continuing medical education and new technologies. Again, it may be assumed that the results overestimate the application of parameters of right ventricular function.

Due to the mode of recruitment this study analyzes a heterogenic sample. Participants were contacted through the network of an online teaching website, including social media platforms. Therefore, the rate of participation in relation to those contacted cannot be determined. Thus, the analyzed group is not a representative sample but rather a large real-life group of echocardiographers. Considering the homogenous results showing a similar distribution of applied methods in all countries, professional groups, and levels of expertise, it can be assumed that the presented data nevertheless represents the real world daily clinical practice.

More information on the reasons for the meager use of modern parameters would have been of great interest. This survey focused on the assessment of the current state of clinical practice, further questions regarding reasons for certain answers were not asked. The nature of the short questionnaire allowed for a large number of participants.

\section{Conclusions}

Eyeballing and TAPSE may lead to misinterpretations of right ventricular function. However, the two parameters are applied most frequently for the quantification of right ventricular function. New methods such as 3D echocardiography and RV-GLS are employed by some physicians, mainly in the university hospital setting. Although these advanced parameters were shown to be highly accurate, they are rarely used in daily clinical routine.

Our investigation revealed that the assessment of right ventricular function by health care professionals does not 
conform to current guidelines. This is especially true of the non-academic setting. It therefore remains the task of the echocardiographic community to continue teaching about the importance of thorough assessment of right ventricular function.

Acknowledgements Open access funding provided by Medical University of Vienna.

\section{Compliance with ethical standards}

Conflict of interest All authors declare that they have no conflict of interest.

Open Access This article is distributed under the terms of the Creative Commons Attribution 4.0 International License (http://creativeco mmons.org/licenses/by/4.0/), which permits unrestricted use, distribution, and reproduction in any medium, provided you give appropriate credit to the original author(s) and the source, provide a link to the Creative Commons license, and indicate if changes were made.

\section{References}

1. Bellsham-Revell HR, Simpson JM, Miller OI, Bell AJ (2013) Subjective evaluation of right ventricular systolic function in hypoplastic left heart syndrome: how accurate is it? J Am Soc Echocardiogr 26(1):52-56

2. Ling LF, Obuchowski NA, Rodriguez L, Popovic Z, Kwon D, Marwick TH (2012) Accuracy and interobserver concordance of echocardiographic assessment of right ventricular size and systolic function: a quality control exercise. J Am Soc Echocardiogr 25(7):709-713

3. Rudski LG, Lai WW, Afilalo J, Hua L, Handschumacher MD, Chandrasekaran K et al (2010) Guidelines for the echocardiographic assessment of the right heart in adults: a report from the American Society of Echocardiography endorsed by the European Association of Echocardiography, a registered branch of the European Society of Cardiology, and the Canadian Society of Echocardiography. J Am Soc Echocardiogr 23(7):685-713 (quiz 86-88)

4. Forfia PR, Fisher MR, Mathai SC, Housten-Harris T, Hemnes AR, Borlaug BA et al (2006) Tricuspid annular displacement predicts survival in pulmonary hypertension. Am J Respir Crit Care Med 174(9):1034-1041

5. Ghio S, Klersy C, Magrini G, D'Armini AM, Scelsi L, Raineri C et al (2010) Prognostic relevance of the echocardiographic assessment of right ventricular function in patients with idiopathic pulmonary arterial hypertension. Int J Cardiol 140(3):272-278

6. Sato T, Tsujino I, Ohira H, Oyama-Manabe N, Yamada A, Ito YM et al (2012) Validation study on the accuracy of echocardiographic measurements of right ventricular systolic function in pulmonary hypertension. J Am Soc Echocardiogr 25(3):280-286

7. Pavlicek M, Wahl A, Rutz T, de Marchi SF, Hille R, Wustmann K et al (2011) Right ventricular systolic function assessment: rank of echocardiographic methods vs. cardiac magnetic resonance imaging. Eur J Echocardiogr 12(11):871-880

8. Sachdev A, Villarraga HR, Frantz RP, McGoon MD, Hsiao JF, Maalouf JF et al (2011) Right ventricular strain for prediction of survival in patients with pulmonary arterial hypertension. Chest 139(6):1299-1309
9. Haeck ML, Scherptong RW, Marsan NA, Holman ER, Schalij MJ, Bax JJ et al (2012) Prognostic value of right ventricular longitudinal peak systolic strain in patients with pulmonary hypertension. Circ Cardiovasc Imaging 5(5):628-636

10. Focardi M, Cameli M, Carbone SF, Massoni A, De Vito R, Lisi M et al (2015) Traditional and innovative echocardiographic parameters for the analysis of right ventricular performance in comparison with cardiac magnetic resonance. Eur Heart J Cardiovasc Imaging 16(1):47-52

11. Anavekar NS, Gerson D, Skali H, Kwong RY, Yucel EK, Solomon SD (2007) Two-dimensional assessment of right ventricular function: an echocardiographic-MRI correlative study. Echocardiography 24(5):452-456

12. Anavekar NS, Skali H, Bourgoun M, Ghali JK, Kober L, Maggioni AP et al (2008) Usefulness of right ventricular fractional area change to predict death, heart failure, and stroke following myocardial infarction (from the VALIANT ECHO Study). Am J Cardiol 101(5):607-612

13. Austin C, Alassas K, Burger C, Safford R, Pagan R, Duello K et al (2015) Echocardiographic assessment of estimated right atrial pressure and size predicts mortality in pulmonary arterial hypertension. Chest 147(1):198-208

14. Peyrou J, Chauvel C, Pathak A, Simon M, Dehant P, Abergel E (2017) Preoperative right ventricular dysfunction is a strong predictor of 3 years survival after cardiac surgery. Clin Res Cardiol 106(9):734-742

15. Blanchard DG, Malouf PJ, Gurudevan SV, Auger WR, Madani MM, Thistlethwaite $P$ et al (2009) Utility of right ventricular Tei index in the noninvasive evaluation of chronic thromboembolic pulmonary hypertension before and after pulmonary thromboendarterectomy. JACC Cardiovasc Imaging 2(2):143-149

16. Singbal Y, Vollbon W, Huynh LT, Wang WY, Ng AC, Wahi S (2015) Exploring noninvasive tricuspid $\mathrm{dP} / \mathrm{dt}$ as a marker of right ventricular function. Echocardiography 32(9):1347-1351

17. Mertens LL, Friedberg MK (2010) Imaging the right ventriclecurrent state of the art. Nat Rev Cardiol 7(10):551-563

18. Surkova E, Muraru D, Iliceto S, Badano LP (2016) The use of multimodality cardiovascular imaging to assess right ventricular size and function. Int J Cardiol 214:54-69

19. Muraru D, Spadotto V, Cecchetto A, Romeo G, Aruta P, Ermacora $D$ et al (2016) New speckle-tracking algorithm for right ventricular volume analysis from three-dimensional echocardiographic data sets: validation with cardiac magnetic resonance and comparison with the previous analysis tool. Eur Heart J Cardiovasc Imaging 17(11):1279-1289

20. Lang RM, Badano LP, Mor-Avi V, Afilalo J, Armstrong A, Ernande L et al (2015) Recommendations for cardiac chamber quantification by echocardiography in adults: an update from the American Society of Echocardiography and the European Association of Cardiovascular Imaging. J Am Soc Echocardiogr 28(1):1-39.e14

21. http://datahelpdesk.worldbank.org/knowledgebase/articles/90651 9

22. Giusca S, Dambrauskaite V, Scheurwegs C, D'hooge J, Claus P, Herbots L et al (2010) Deformation imaging describes right ventricular function better than longitudinal displacement of the tricuspid ring. Heart 96(4):281-288

23. Tamborini G, Muratori M, Brusoni D, Celeste F, Maffessanti $\mathrm{F}$, Caiani EG et al (2009) Is right ventricular systolic function reduced after cardiac surgery? A two- and three-dimensional echocardiographic study. Eur J Echocardiogr 10(5):630-634

24. Soslow JH, Usoro E, Wang L, Parra DA (2016) Evaluation of tricuspid annular plane systolic excursion measured with cardiac MRI in children with tetralogy of Fallot. Cardiol Young 26(4):718-724 
25. Hsiao SH, Lin SK, Wang WC, Yang SH, Gin PL, Liu CP (2006) Severe tricuspid regurgitation shows significant impact in the relationship among peak systolic tricuspid annular velocity, tricuspid annular plane systolic excursion, and right ventricular ejection fraction. J Am Soc Echocardiogr 19(7):902-910
26. Park JB, Lee SP, Lee JH, Yoon YE, Park EA, Kim HK et al (2016) Quantification of right ventricular volume and function using single-beat three-dimensional echocardiography: a validation study with cardiac magnetic resonance. J Am Soc Echocardiogr 29(5):392-401 